\title{
The bacterial protein toxin, cytotoxic necrotizing factor 1 (CNF1) provides long-term survival in a murine glioma model
}

\author{
Eleonora Vannini ${ }^{1,2+}$, Anna Panighini ${ }^{1 \dagger}$, Chiara Cerri $^{1}$, Alessia Fabbri ${ }^{3}$, Simonetta Lisi ${ }^{2}$, Enrico Pracucci ${ }^{2}$,
} Nicola Benedetto ${ }^{4}$, Riccardo Vannozzi ${ }^{4}$, Carla Fiorentini ${ }^{3}$, Matteo Caleo ${ }^{1 *+}$ and Mario Costa ${ }^{1 \dagger}$

\begin{abstract}
Background: Glioblastomas are largely unresponsive to all available treatments and there is therefore an urgent need for novel therapeutics. Here we have probed the antineoplastic effects of a bacterial protein toxin, the cytotoxic necrotizing factor 1 (CNF1), in the syngenic GL261 glioma cell model. CNF1 produces a long-lasting activation of Rho GTPases, with consequent blockade of cytodieresis in proliferating cells and promotion of neuron health and plasticity.

Methods: We have tested the antiproliferative effects of CNF1 on GL261 cells and human glioma cells obtained from surgical specimens. For the in vivo experiments, we injected GL261 cells into the adult mouse visual cortex, and five days later we administered either a single intracerebral dose of CNF1 or vehicle. To compare CNF1 with a canonical antitumoral drug, we infused temozolomide (TMZ) via minipumps for 1 week in an additional animal group.

Results: In culture, CNF1 was very effective in blocking proliferation of GL261 cells, leading them to multinucleation, senescence and death within 15 days. CNF1 had a similar cytotoxic effect in primary human glioma cells. CNF1 also inhibited motility of GL261 cells in a scratch-wound migration assay. Low dose (2 nM) CNF1 and continuous TMZ infusion significantly prolonged animal survival (median survival 35 days vs. 28 days in vehicle controls). Remarkably, increasing CNF1 concentration to $80 \mathrm{nM}$ resulted in a dramatic enhancement of survival with no obvious toxicity. Indeed, $57 \%$ of the CNF1-treated animals survived up to 60 days following GL261 glioma cell transplant.

Conclusions: The activation of Rho GTPases by CNF1 represents a novel potential therapeutic strategy for the treatment of central nervous system tumors.
\end{abstract}

Keywords: Glioma, Mouse, Cerebral cortex, CNF1, Temozolomide

\section{Background}

Gliomas are primary central nervous system tumors that arise from astrocytes, oligodendrocytes or their precursors. Following the World Health Organization (WHO) classification, gliomas can be classified in 4 groups according to their histological characteristics and the most malignant form is glioblastoma multiforme (GBM). GBM is uniformly fatal and largely unresponsive to all available treatments. Despite intensive therapy including surgery, radiotherapy and chemotherapy, the average of survival of patients with glioblastoma usually is 15 months from the time of first

\footnotetext{
*Correspondence: caleo@in.cnr.it

${ }^{\dagger}$ Equal contributors

${ }^{1}$ CNR Neuroscience Institute, Via Moruzzi 1, 56124 Pisa, Italy

Full list of author information is available at the end of the article
}

diagnosis [1,2]. Conventional surgical excision, generally limited to the main tumor mass, does not remove the microscopic foci of neoplastic cells that invade the surrounding normal brain substance beyond the main tumor mass, and that are responsible for the inevitable tumor recurrence. Radiotherapy and chemotherapy, often associated to surgery, cannot ablate completely these tumors, since this would require unacceptably high radiation/chemotherapic doses that result in severe brain-neuron damage. There is therefore a clear need to accelerate progress in the development of new strategies for treatment of glioma.

Several therapeutic approaches for glioma are currently being investigated in animal models and patients. They include delivery of cytotoxic genes and proteins to glioma 
cells, suppression of angiogenesis, and immune stimulation [3]. Concerning chemotherapy, alkylating agents such as temozolomide (TMZ) are widely used in the treatment of brain tumours [4]. As a cytotoxic alkylating agent, TMZ is converted at physiologic $\mathrm{pH}$ to the short-lived active compound, monomethyl triazeno imidazole carboxamide (MTIC). The cytotoxicity of MTIC is primarily due to methylation of DNA at the $\mathrm{O} 6$ and $\mathrm{N} 7$ positions of guanine, resulting in inhibition of DNA replication. Chemotherapics have substantial side effects and limited efficacy, and this further underlies the need of innovative approaches for glioma treatment.

In this paper we describe a potential novel therapy for glioma, based on intracerebral administration of cytotoxic necrotizing factor 1 (CNF1), a bacterial protein toxin produced by specific strains of Escherichia coli. CNF1 is a single-chain protein, consisting of a Nterminal domain involved in cell binding, a middle region mediating membrane translocation, and a $\mathrm{C}$ terminal catalytic domain. The C-terminal part of CNF1 is released into the cytosol where it catalyzes the deamidation of a single glutamine residue of the Rho GTPases (RhoA, Rac1 and Cdc42). Rho GTPases are molecular switches that cycle between a GDP-bound inactive and a GTP-bound active state to control a multitude of cellular events, like actin cytoskeleton organization as well as gene transcription, cell proliferation, and survival [5]. Rho GTPases deamidated by CNF1 are not able to hydrolyse GTP and remain in a persistent activated state $[6,7]$ which is followed by partial deactivation of these regulatory proteins via degradation by the ubiquitin-proteasome pathway [8]. The persistent activation of Rho GTPases by CNF1 causes a remarkable reorganization of the actin cytoskeleton with dramatic functional consequences. In particular, cultured proliferating cells exposed to CNF1 acquire a multinucleated phenotype (cytotoxic effect), due to stabilization of the actin network and prevention of cytodieresis despite ongoing nuclear division [9]. On the other hand, our recent studies have demonstrated "plasticizing" effects of CNF1 in neurons. Specifically, intracerebral administration of CNF1 improves neuronal function, learning and memory $[10,11]$, and these effects are associated with a enhancement of brain plasticity, exemplified by the increase in spine density in cortical neurons [10].

In view of these striking, differential effects of CNF1 on proliferating cells and neurons, we have probed for the first time the potential antitumoral effects of this toxin in cell culture and in an animal model. Among the available glioma models, we adopted the well accepted GL261 syngeneic mouse model of high grade glioma [12,13] based on intracerebral injection of GL261 cells in C57/Bl6 mice. We demonstrate the cytostatic and cytotoxic effects of CNF1 on GL261 glioma cell line, and the survival-promoting effect on tumor-bearing animals. We have also provided proof-of-principle for cytotoxic effects of CNF1 in human tumor cells.

\section{Methods}

\section{GL261 glioma cell cultures}

The murine glioma GL261 cell line was a kind gift from Dr. C. Sala (CNR Neuroscience Institute, Milan). GL261 cells were grown in complete Dulbecco's modified Eagle's medium (DMEM) containing 10\% Newborn calf serum, $4.5 \mathrm{~g} / \mathrm{L}$ glucose, $2 \mathrm{mM}$ glutamine, $100 \mathrm{UI} / \mathrm{ml}$ penicillin and $100 \mathrm{mg} / \mathrm{ml}$ streptomycin at $37^{\circ} \mathrm{C}$ in $5 \%$ $\mathrm{CO}_{2}$ with media changes three times per week.

\section{CNF1}

E. coli CNF1 was obtained from the 392 ISS strain (kindly provided by V. Falbo, Istituto Superiore di Sanità, Rome, Italy) and purified as described previously [14]. The levels of lipopolysaccharide (LPS) in the CNF1 preparation were assessed by the Limulus Amebocyte Lysate (LAL) kinetic chromogenic assay (performed by LONZA Verviers Sprl). The LPS concentration determined by the assay $(0.07 \mathrm{ng} / \mathrm{ml})$ was much lower than that required to achieve biological effects (e.g. $1 \mathrm{ng} / \mathrm{ml}$ in macrophages, one of the most sensitive cells to LPS).

The activity of every batch of CNF1 was tested on GL261, treating the cells for 24 hours. Three parameters were considered: i) cells morphology (enlargement and flattening of cells), ii) increased size of nucleoli and iii) the ratio between mono and multinucleated cells. The activity of each CNF1 preparation was considered satisfactory if at least one of the three parameters indicated above were present in more than $60 \%$ of treated cells.

\section{Clonogenic assay}

GL261 cells were harvested by trypsinization, counted and seeded onto twenty four-well plates at a density of 300 cells/plate. To assess the effect of CNF1 on cell proliferation, GL261cells were incubated for 9 days with different concentrations of CNF1 (from $8 \times 10^{-11}$ to $3.2 \times$ $\left.10^{-9} \mathrm{M}\right)$. Nine days after treatment, cells were stained with crystal violet, the number of colonies containing at least 50 cells was counted [15] and the effective half inhibitory dose (IC50) of the toxin was calculated on the basis of linear regression equation.

\section{Wound healing-migration assay}

The wound healing migration assay was performed according to Liang [16] with minor modifications. Briefly, GL261 cells were seeded in 6-well cell plates and cultured to a confluent monolayer. A sterile pipette tip $(200 \mu \mathrm{l})$ was used to make a straight scratch on the monolayer of cells and the wound was allowed to heal for 8, 24 and 48 hours. To evaluate CNF1 effects, GL261 cells were incubated with CNF1 for 24 hours before 
making the scratch, and wound healing was assessed 8, 24 and 48 hours after the injury. The cells were then fixed with methanol and stained with crystal violet. The extent of cell migration was photographed and the wound size measured using image analysis software (ImageJ).

\section{Apoptosis-necrosis assays}

Apoptotic and/or necrotic cells were determined using Annexin V- Propidium Iodide (PI) Staining Kit. The assay was performed following the manufacturer instructions (Annexin V kit, BD Pharmingen). Briefly, 300 GL261 cells were seeded on twenty four-well plates and incubated in CNF1 (18 nM) for 10 days. Annexin V and Propidium Iodide were diluted 1:200 in KREB medium $\left(\mathrm{NaCl} 120 \mathrm{mM}, \mathrm{NaKCO}_{3} 25 \mathrm{mM}, \mathrm{KCl} 4.7 \mathrm{mM}, \mathrm{KH}_{2} \mathrm{PO}_{4}\right.$ $1.18 \mathrm{mM}, \mathrm{MgSO}_{4} 1.18 \mathrm{mM}, \mathrm{CaCl}_{2} 2.5 \mathrm{mM}$, EDTA $0.026 \mathrm{mM}$, glucose $5.5 \mathrm{mM}$ ). Cells were also stained with Hoechst dye (bisbenzimide, Sigma; 1:500 in PBS) and counted with fluorescence microscopy. We classified cells as positive for annexin $\mathrm{V}$ only (Ann V+PI-), positive for PI only (Ann V- PI+), positive for both markers (Ann V + PI+) or unlabeled.

\section{Senescence-Associated Beta-Galactosidase (SA beta-gal) Assay}

To determine cellular senescence, GL261 cells were plated in triplicate at low density (50\% confluence) in 24-well plates. The cells were treated with CNF1 (1 nM) and incubated for 24,48 or 72 hours before betagalactosidase measure (senescence detection kit, Abcam catalog ab65351). The percentage of positively stained cells was determined after counting three random fields of cells. Representative microscopic fields were photographed under a $20 \times$ objective.

\section{Human glioblastoma cell cultures}

Human biopsies of glioblastoma multiforme (GBM) were collected from 2 subjects who underwent brain surgery for tumor removal. The study was approved by the Human Ethics Committee of the University of Pisa and Pisa Hospital. Written, informed consent was obtained from the patients according to institutional guidelines. Patient 1 had a left parietal lesion resembling a high grade glioma on a contrast enhanced MRI scan, while patient 2 had a similar lesion located in the left fronto-parietal region. A surgical gross total resection was performed and the histological examination confirmed the suspected tumor type (WHO grade IV) in both cases. After collection, primary tumor cells were isolated according to [17]. Briefly, tissue explants were incubated with trypsin in DMEM for 10 minutes at $37^{\circ} \mathrm{C}$ and then added of 3 volumes of DMEM with 10\% FBS. Tissue was completely disgregated by gentle pipetting.
Cells suspension was then plated on tissue culture dishes and culture medium (DMEM, 10\%FBS, $100 \mathrm{IU} / \mathrm{ml}$ penicillin,100 $\mathrm{mg} / \mathrm{ml}$ streptomycin) was replaced every 3-5 days. After a week cells were trypsinized and splitted. At passage five [18] cells were plated on cover slips and then treated with $18 \mathrm{nM}$ CNF1 for 9 days. At this time point, cells were fixed and stained to observe their morphology.

\section{Animals and tumor induction}

To induce glioma formation, C57BL/6 mice (12-14 weeks old) received a stereotaxically guided injection of 40,000 GL261 cells $(20,000$ cells/ $\mu$ l PBS solution) into the visual cortex $(2 \mathrm{~mm}$ lateral to the midline and in correspondence with lambda) using fine glass micropipettes (tip diameter $40 \mu \mathrm{m})$. All experimental procedures were in conformity to the European Communities Council Directive 86/609/EEC. The animal experiments described in this manuscript have been approved by the Italian Ministry of Health, Department of Animal Health (Office n. 6), with decree \#2582012/B, dated Oct 23, 2012.

\section{CNF1 injections and TMZ minipumps}

Five days after GL261 cells injection (tumor induction), mice were divided into three groups. The first group received CNF1 injection, the second Tris- $\mathrm{HCl}$ buffer injection (control condition). Stereotaxic infusions of CNF1/Tris- $\mathrm{HCl}(1 \mu \mathrm{l}$ of a 2 or $80 \mathrm{nM}$ solution) were made into the primary visual cortex of adult mice under avertin anesthesia (intraperitoneal injection of 2,2,2-tribromoethanol solution; $0.1 \mathrm{ml} / 5 \mathrm{~g}$ body weight). Injections were performed in three sites: $1.5,2$ and $2.5 \mathrm{~mm}$ lateral to the midline in correspondence with lambda. $\mathrm{CNF} 1 /$ Tris- $\mathrm{HCl}$ injections was slowly delivered at a depth of $0.7-0.8 \mathrm{~mm}$ from the pial surface.

The third experimental group received Temozolomide (TMZ) micropumps implantation for a week. Minipump implantation was performed as described previously $[19,20]$. Micro-osmotic pumps (Alzet 1007D; Alza, USA; pumping rate $0.5 \mu \mathrm{l} / \mathrm{hr}$ ) were filled with TMZ (20, 140 and $200 \mu \mathrm{M}$ solution) and connected with polyethylene tubing to $30 \mathrm{G}$ stainless steel cannulae [20]. A small hole was made in the skull ( $2 \mathrm{~mm}$ lateral, $1 \mathrm{~mm}$ anterior) and the cannula was lowered into the cortex. The minipump was positioned subcutaneously under the neck and the cannula was secured to the skull with acrylic cement. Animals survival was checked daily and monitored up to 60 days after tumor implantation. At this time, mice that were still alive were deeply anesthetized and perfused with $4 \%$ paraformaldehyde. Coronal brain sections through the occipital cortex (45 $\mu$ m thick) were cut with a freezing microtome, stained with Hoechst dye (1:500, Sigma) and histopatological examinations were carried out. 


\section{Statistical analysis}

Statistical analysis was performed with SigmaPlot (version 11). Differences between three or more groups were evaluated with one way analysis of variance (ANOVA) followed by Holm-Sidak test.

Survival analysis was performed using Kaplan-Meier (LogRank) statistics.

\section{Results}

\section{CNF1 blocks GL261 cell proliferation in culture}

We first used a clonogenic assay to determine whether glioma cell proliferation is affected by CNF1. GL261 cells were plated at low density and exposed to different concentrations of CNF1. Colonies were counted after 9 days. We found that CNF1 inhibits colony formation in a dose-dependent manner (Figure 1). The half maximal inhibitory concentration (IC50) of CNF1 was found to be $0.47 \mathrm{nM}$ (Figure 1B). In subsequent experiments, we always used CNF1 concentrations in the nanomolar range to ensure robust effects on cell proliferation.

\section{A short exposure time is sufficient for CNF1 to exert its effects}

In order to determine the minimal exposure time required by CNF1 to exert its effects, cells were treated with CNF1 (18 nM) for different times (ranging from 1 up to 16 hours). At the end of each incubation time, the

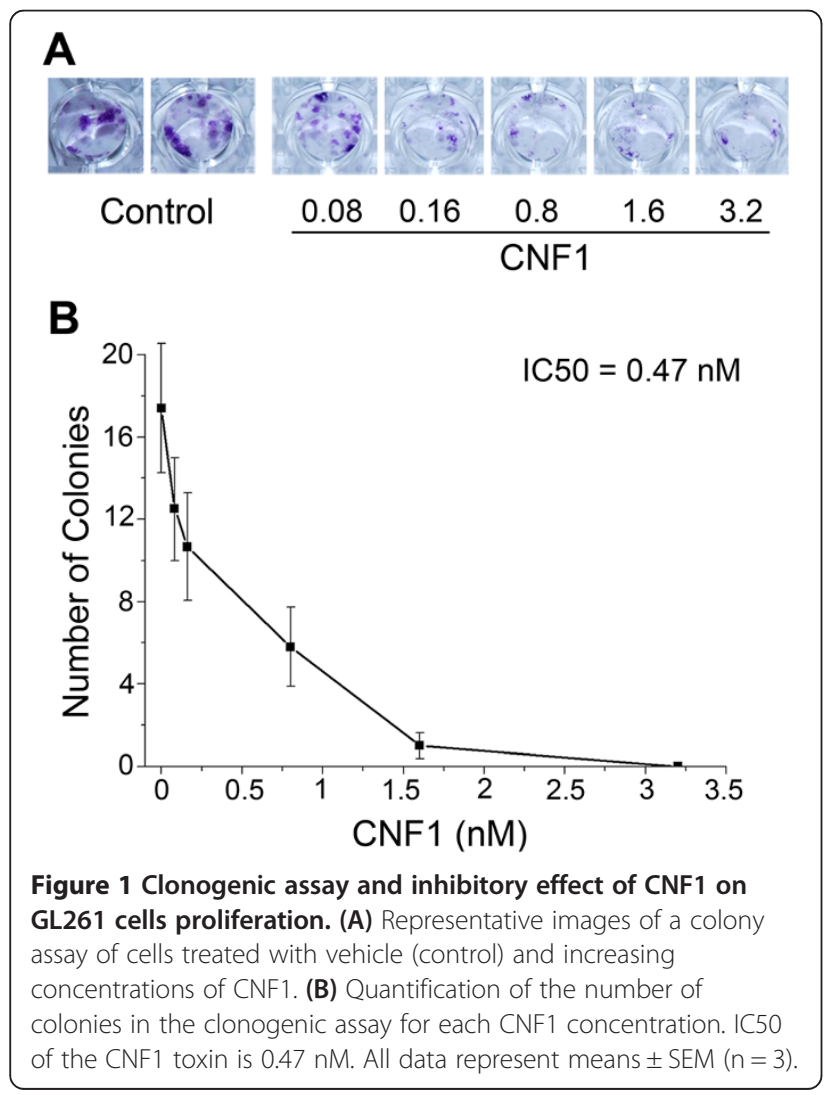

toxin was replaced with fresh medium. Effects of the toxin were evaluated by counting cell numbers five days after treatment. The experiments demonstrated that 1 hour exposure to CNF1 was sufficient to stop proliferation, as shown by the dramatic (about $80 \%$ ) reduction in cell numbers as compared to control (no treatment) conditions (one way ANOVA, $\mathrm{p}<0.001$; post hoc HolmSidak test, $\mathrm{p}<0.001$; Figure 2A). CNF1 effects became even more prominent by increasing the exposure time to 16 hours ( $<<0.001$; Figure $2 \mathrm{~A}$ ). We also evaluated the morphological appearance of the treated cells (Figure 2B). We found that in control conditions all cells were mononucleated, while in cells treated with CNF1 for 16 hours this phenotype decreased to $16 \%$, and the percentage of multinucleated cells increased with longer CNF1 exposures (one way ANOVA, $\mathrm{p}<0.001$; post hoc HolmSidak test, $\mathrm{p}<0.001$; Figure $2 \mathrm{C}$ ).

\section{CNF1 reduces the migration capability of GL261 cells}

A wound healing assay was performed in the GL261 cells incubated in culture medium with or without CNF1 (1 nM) to observe the effect of CNF1 on the migration. The area that the cells had migrated within 8 , 24 and 48 hours (toward the initially scratched midline, from the border line) was measured. The experiments demonstrated that after 8 hours the number of cells migrating in the wound increased in normally growing cells, whereas fewer cells migrated in the wound area in CNF1-treated cells. The treatment effect was more evident after 24 hours, when in control cells the wound was healed to $80 \%$ whereas in CNF1-treated cells it was closed to about $30 \%$ and, at 48 hours, in control the wound was totally closed while in treated cells was half closed (Figure 3A and B).

\section{Fate of GL261 cells after prolonged CNF1 exposure}

To determine the fate of polynucleated GL261, cells were treated with CNF1 (18 nM) at day one and then cultured without removal of the toxin for 15 days. We found a clear cytostatic effect of CNF1 until day 12 (Figure 4A). At this time point, GL261 cells treated with CNF1 still showed the characteristic altered morphology (enlargement and flattening of cells, increased size of nucleoli and multinucleation; see also Methods; Figure 4A, inset). Importantly, treatment for 15 days resulted in no survival of GL261 cells (Figure 4A), consistent with a cytotoxic action of CNF1.

As a first step towards understanding the mechanisms of CNF1-induced GL261 cell death, we stained cells with markers of apoptosis and necrosis (annexin $\mathrm{V}$ and propidium iodide, respectively) 10 days after treatment. We chose this time point as it corresponds to a stage that just precedes cell disappearance, however all cells are still attached to dish and show the morphological 


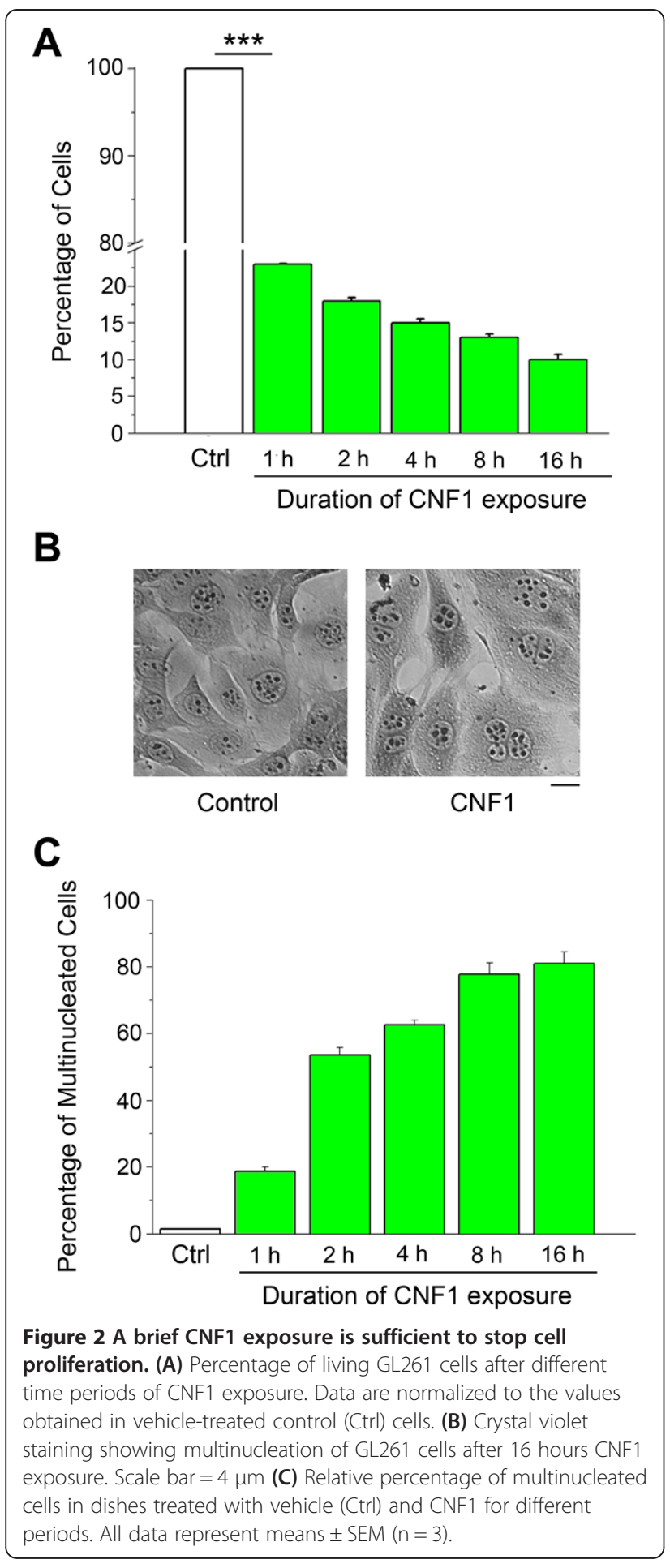

features of senescence. As a positive control for apoptosis, we used GL261 cells deprived of serum for 2 days, which showed a robust increase in annexin V, but not PI staining (Figure 4B, left columns, AnnV + PI-; one way ANOVA followed by Holm-Sidak test, $\mathrm{p}<0.05$; Figure 4B, grey bars). GL261 cells treated with CNF1 for 10 days showed no clear evidence for either apoptotic (Figure 4B, left columns, "AnnV + PI-") or necrotic cell death (Figure 4B, "AnnV- PI+"). However, more than 50\% of CNF1treated cells at 10 days resulted positive for both markers (Figure 4B, "AnnV + PI+"; one way ANOVA followed by Holm-Sidak test, $\mathrm{p}<0.05)$ indicating profound cellular distress and late stages of cell demise.

\section{CNF1 induces early activation of senescence pathway}

As indicated above, the GL261 cells treated with CNF1 are arrested in the cell proliferation and show a senescent morphology (enlargement and flattening of cells). We therefore adopted the $\beta$-Galactosidase activity, as a well accepted senescence marker, to confirm our morphological observation. The blue $\beta$-Galactosidase staining in treated cells was detectable as early as one day after treatment with the toxin $(1 \mathrm{nM})$, and became intense and expressed in every cell of the culture after 72 hours. The SA- $\beta$-gal staining was not detected or barely detected in untreated control cells (Figure 5).

\section{CNF1-induced multinucleation in human primary tumoral cells}

To obtain proof-of-principle for CNF1 action on human glioma cells, two surgical specimens were obtained from patients with GBM (WHO grade IV). CNF1 effects (18 $\mathrm{nM}$ ) were examined in early passages (five passages) cell lines from these primary GBM specimens. Similar to GL261 cells, we observed the CNF1-induction of multinucleation in human tumor cells treated with the toxin for 9 days (Figure 6).

Overall, these data indicate a powerful cytostatic and cytotoxic effect of CNF1 on cultured glioma cells, including tumor cells from human subjects.

\section{Intracerebral CNF1 treatment enhances survival in a murine glioma model}

Prompted by the cytostatic and cytotoxic effects of CNF1 in vitro, we tested the actions of this toxin after intracerebral inoculation of GL261 cells in adult mice. The murine GL261 model is a well accepted and widely used syngeneic transplant model for experimental glioma tumors, and reproduces many of the histopathological features of human glioma $[12,13]$.

GL261 cells $(40,000$ cells in $2 \mu \mathrm{l})$ were transplanted at the level of layer VI into the occipital (visual) cortex of adult mice. Five days later, animals were injected around the transplant site with either vehicle (TRIS buffer; $\mathrm{n}=$ 19) or CNF1 (2 nM; $n=9$; Figure 7A top). This dose of CNF1 has been previously shown to trigger a prolonged activation of Rho GTPases (particularly, Rac1) in vivo $[10,11]$. Kaplan-Meier survival analysis indicated a clear survival-promoting effect of CNF1 when compared to vehicle ( $\log$ Rank test, $\mathrm{p}=0.02$; Figure $7 \mathrm{~B}$ ). 


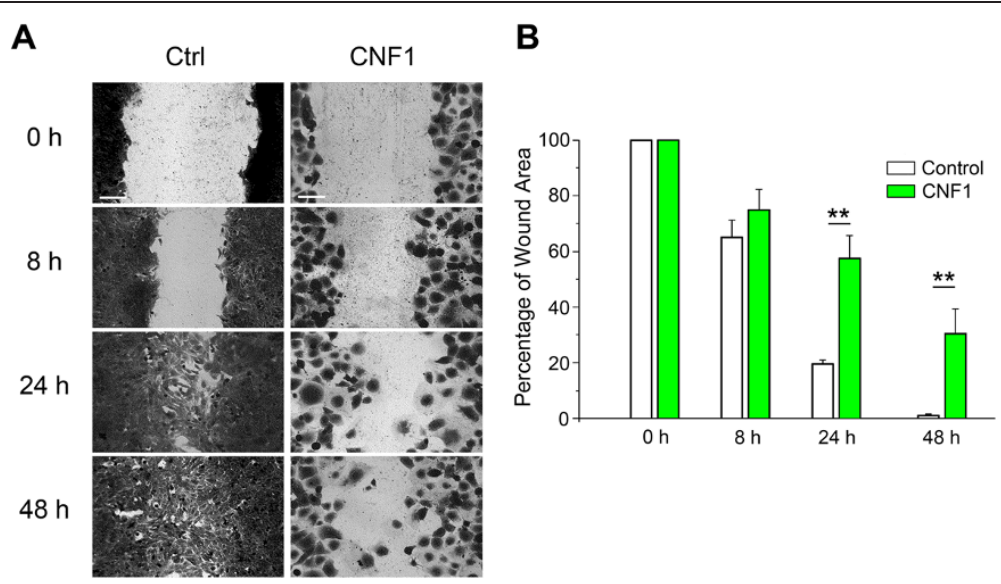

Figure 3 Wound-healing migration assay of CNF1-treated GI261 cells. (A) Representative images of wound in untreated cells (left column) and CNF1-treated cells (right column) at different time points (0,8,24 and 48 hours). The data demonstrate the inability of CNF1-treated cells to invade the wound area. (B) Quantitative plots of the wound size at different time points in the two conditions (CNF1 and control). Data shown are representative of three independent experiments. All data represent means \pm SEM. (two-way ANOVA, ${ }^{* *} p<0.001$ ). Scale bar $=100 \mu \mathrm{m}$.

Temozolomide effect on survival of glioma-bearing mice To directly compare the actions of CNF1 with those of classical chemotherapy, we used continuous intracerebral minipump infusions (from day 5 to day 12 post-GL261 transplant) of the alkylating agent TMZ (Figure 7A, bottom). The infusion cannula was placed directly into the cortex at a distance of $1 \mathrm{~mm}$ from the transplant site. Initial experiments indicated that doses of TMZ higher than $200 \mu \mathrm{M}$ were toxic for the animals, as shown by weight loss and high mortality rate. We therefore selected a concentration of $140 \mu \mathrm{M}$ TMZ for the minipump experiments. We found that TMZ significantly prolonged the length of survival of glioma-bearing mice (Figure 7B). In particular, the statistical analysis indicated that $2 \mathrm{nM}$ CNF1 and $140 \mu \mathrm{M}$ TMZ enhanced animal survival to a similar extent (Log Rank test, $\mathrm{p}=0.003$, followed by Holm-Sidak test; $2 \mathrm{nM}$ CNF1 vs. vehicle, $\mathrm{p}=0.02 ; 140 \mu \mathrm{M}$ TMZ vs. vehicle, $\mathrm{p}=0.002$; 2 nM CNF1 vs. $140 \mu \mathrm{M}$ TMZ, $\mathrm{p}=0.91$; Figure $7 \mathrm{~B})$. Lower doses of TMZ $(20 \mu \mathrm{M})$ were completely ineffective (data not shown; $\mathrm{n}=4$ ).

\section{Higher CNF1 doses produce a dramatic increase in survival of glioma-bearing mice}

We next asked whether an increase in the CNF1 dose would be effective in further enhancing animal survival. To this aim, glioma-bearing mice received a single treatment with CNF1 (80 nM; $\mathrm{n}=7$ ) five days after GL261 cell inoculation. We found a dramatic increase in survival, with $57 \%$ of the animals injected with $80 \mathrm{nM}$ CNF1 still alive 60 days after GL261 cell inoculation (Kaplan-Meier survival analysis, CNF1 $80 \mathrm{nM}$ vs. vehicle, Log Rank test, $\mathrm{p}<0.001$; Figure 8A). An histopathological analysis was conducted in the CNF1-treated glioma-bearing animals surviving over 60 days. We found a small tumor located in the deep cortical layers and surrounded by apparently healthy cortical tissue (Figure 8B). These data indicate the potential of CNF1 in halting glioma growth and preserving neuronal structure.

\section{Discussion}

The data reported in this manuscript demonstrate the therapeutic potential of a bacterial protein toxin, CNF1, in blocking proliferation of glioma cells and prolonging the survival of glioma-bearing mice. At present, there are several evidences on the use of targeted toxins to treat cancer [21]. However, the clinical efficacy of toxins has mainly been observed in hematological malignancies, but not in solid tumors, including GBM. The toxin used in this report, CNF1, is produced by certain pathogenic strains of $E$. Coli and consists of a N-terminal binding domain that interacts with membrane receptors on target cells, a middle translocation domain to enter the cytosol and a C-terminal catalytic domain [22,23]. The catalytic moiety of CNF1 activates members of the Rho GTPase family (Rho, Rac and Cdc42) by conversion (deamidation) of a single glutamine residue into glutamic acid. This aminoacid change impairs GTP hydrolysis thus locking the Rho family proteins in their GTP-bound, active state [22,23]. Depletion of activated Rho GTPases is then accomplished via ubiquitinmediated proteasomal degradation [8].

Since Rho GTPases regulate the dynamics of the actin cytoskeleton, their activation by CNF1 triggers a rapid reorganization of F-actin [24]. In particular, proliferating cells exposed to CNF1 acquire a multinucleated phenotype, due to the inhibition of cytokinesis despite ongoing nuclear division $[22,25,26]$. We have observed CNF1induced multinucleation in either GL261 glioma cells or 


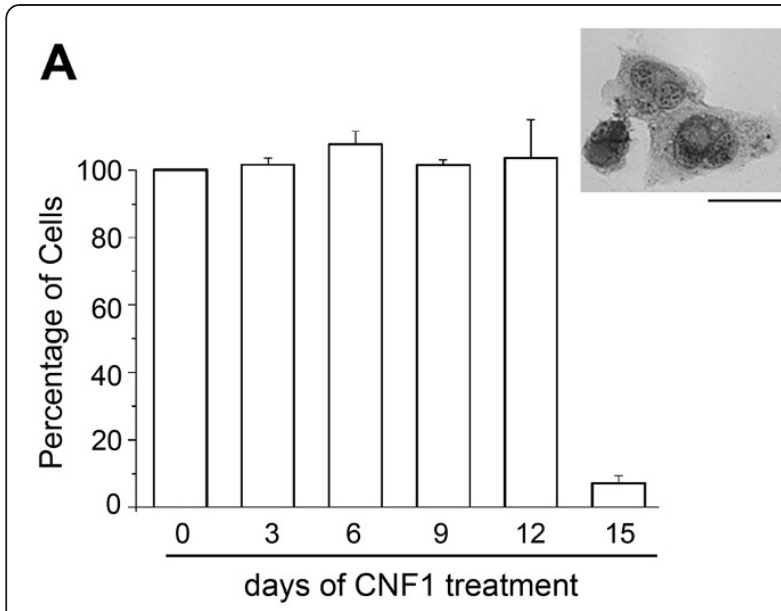

B
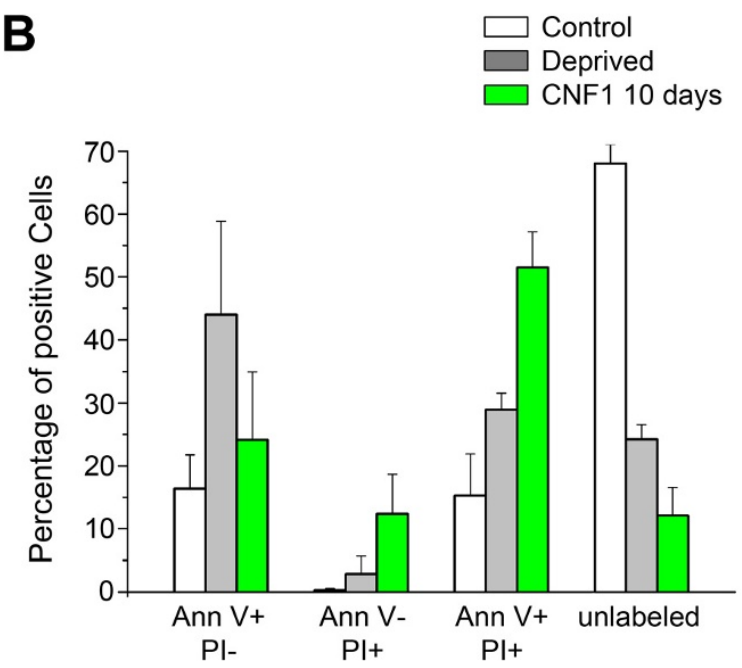

Figure 4 Cytotoxic effect of CNF1 on GL261 cells. (A) Percentage of living GL261 cells after continuous exposure to CNF1 (18 nM). Data represent means \pm SEM $(n=3)$. Inset: Morphology of GL261 cells treated with CNF1 for 9 days. Scale bar $=5 \mu \mathrm{m}$. (B) Percentage of cells stained only by Annexin $\mathrm{V}$ (Ann V + Pl-, left columns), stained only by Propidium lodide (Ann V-PI+), positive for both markers (Ann $\mathrm{V}+\mathrm{PI}+$ ) and unlabeled (right columns). The analysis was performed in untreated GL261 cells (Control, open bars), in GL261 cells serum deprived for 2 days (grey bars), and in GL261 cells treated with CNF1 for 10 days (green bars). CNF1 induces a very significant upregulation in the frequency of Annexin V-Propidium lodide double labelled cells (one way ANOVA followed by Holm-Sidak test, $\mathrm{p}<0.05)$.

early passage cell lines from primary GBM specimens. We have also shown that these multinucleated cells degenerate within about 15 days in vitro.

These data raise the still open question of the identification of the pathway(s) by which CNF1 causes cell degeneration. Experiments with $\beta$-Galactosidase, Annexin V and Propidium Iodide labelling allow us to conclude that senescence and then necrosis account for CNF1-induced GL261 cell death. This is consistent with the senescent-like phenotype (cell enlargement, flattening, increase in size of nuclei and nucleoli) assumed by cells treated with CNF1 (see
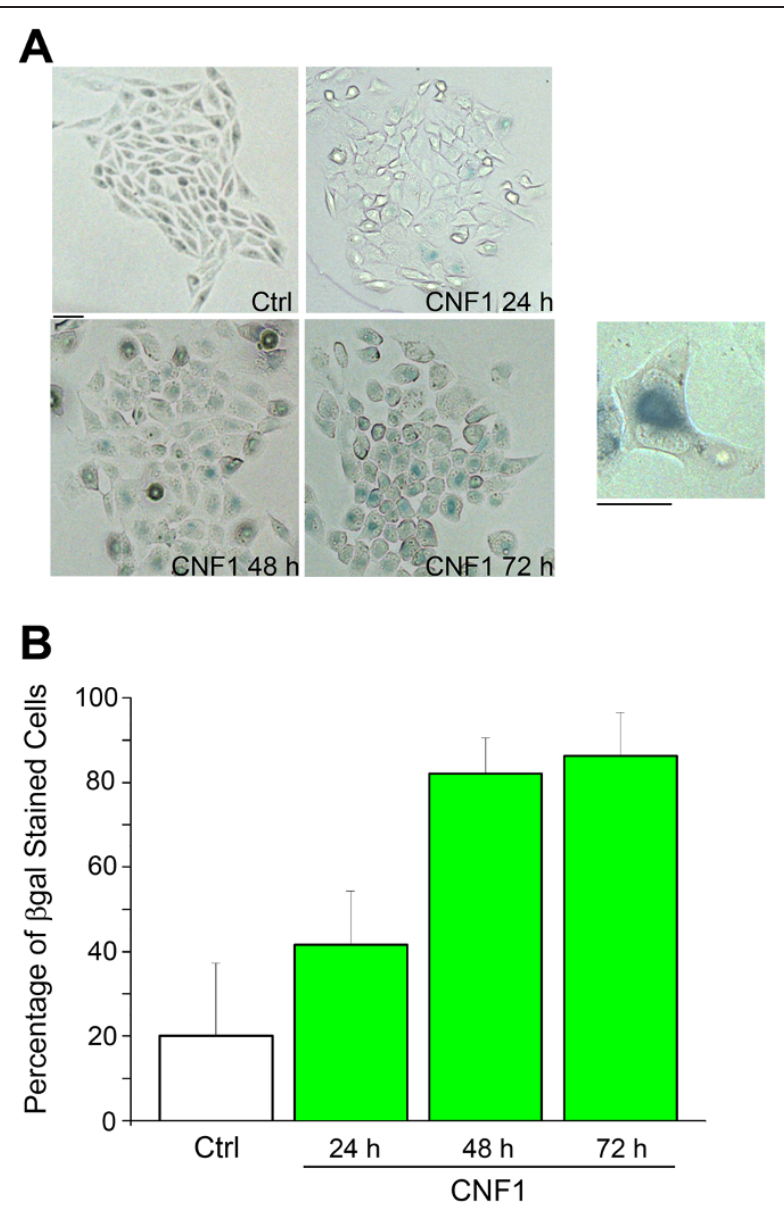

Figure 5 CNF1 induces senescence in GL261 cells: SA-beta-gal staining. (A) Representative images of SA-beta-gal staining of GL261 cells after $24 \mathrm{~h}, 48 \mathrm{~h}$ and $72 \mathrm{~h}$ of CNF1 treatment. All images were taken at 20x magnification. Scale bar $=10 \mu \mathrm{m}$. Inset: representative high magnification of a CNF1-treated cell at 48 hours. Scale bar $=5 \mu \mathrm{m}$. (B) Percentage of beta-gal positive cells at the different time points. Data shown are representative of three independent experiments (one way ANOVA, $p<0.0001$ ). All data represent means \pm SEM.

Figure 4A, inset). At the moment, we cannot exclude the possibility that the autophagy pathway contributes to the CNF1-induced phenotype. A recent paper indicates the activation of the autophagy pathway following the treatment of glioma cells with pertussis toxin and TMZ [27]. Future studies are needed to clarify the possible role of autophagy in the antitumoral action of CNF1.

Since CNF1 is derived from E. coli, it might be argued that contamination by bacterial products (such as LPS) could contribute to the observed antineoplastic effects via the activation of immune responses in the brain [28]. However, this hypothesis is very unlikely, as the amount of LPS is our CNF1 preparation was found to be extremely low, in a range unable to activate macrophages (see Methods).

In GBM and in experimental glioma models, the activation of Rho GTPases (such as Rac1) has been linked 


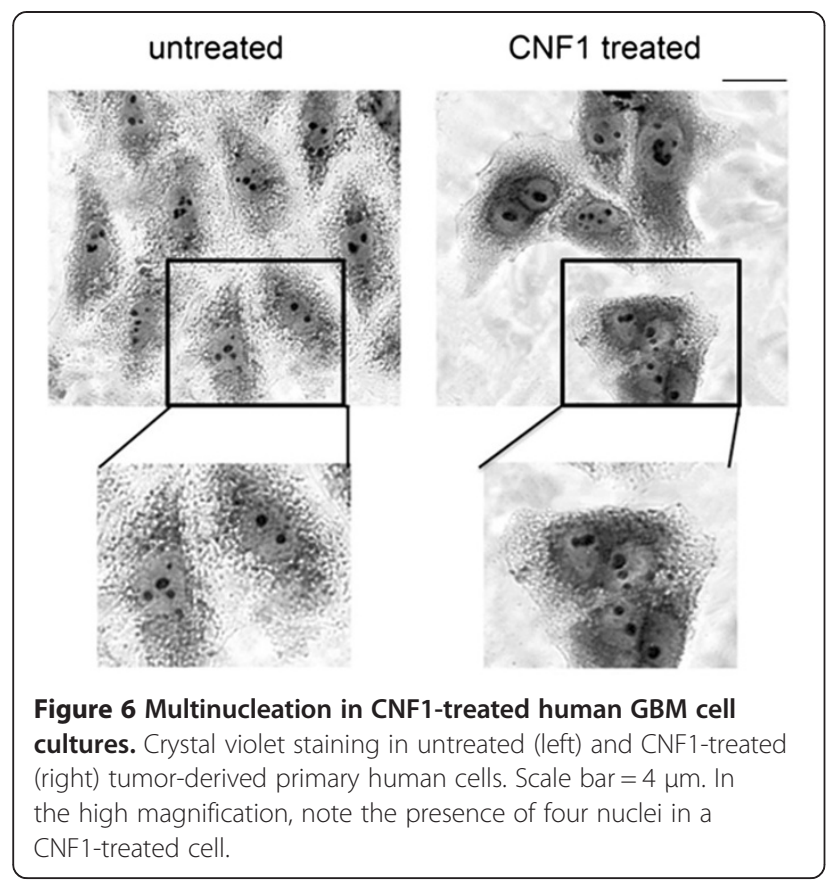

to increased cell invasion [29], pointing to these molecules as key therapeutic targets. In this scenario, the dramatic effect of CNF1 on the actin cytoskeleton reorganization renders cells virtually immobile, and this might substantially enhance the anti-tumoral properties of the toxin. Indeed, we have demonstrated, in the wound migration assay, that CNF1 dramatically decreased the motility of GL261 cells, suggesting a further potential therapeutic feature of this toxin for its possible application in the treatment of glioma tumors. This aspect is important in the context of glioma treatment, because glioma cells tend to diffuse profusely into adjacent healthy tissue. It is well established that CNF1 causes assembly of F-actin in prominent stress fibers and extreme flattening of the cell body $[6,24]$. Since cells in motion need actin dynamics to attach and detach from the extracellular matrix, actin polymerization by CNF1 effectively renders cells stationary.

Furthermore, it is worth noting that the other key aspect of the action of CNF1 is on neuron plasticity and health [10]. This cooperates with the antitumoral effect (reduced proliferation and motility) and may lead to better preservation of neuronal function in the brain areas surrounding the tumor. Experiments to address CNF1mediated functional sparing in glioma models are currently ongoing in our laboratory.

Importantly, a key feature of CNF1 is the rapidity of its action. We found that exposure to CNF1 for $1 \mathrm{hr}$ was sufficient to halt proliferation of most of the treated cells (Figure 2). Since CNF1 is an enzyme, entry of a few toxin molecules inside a cell can lead to the modification of several Rho GTPase targets, providing a dramatic amplifying

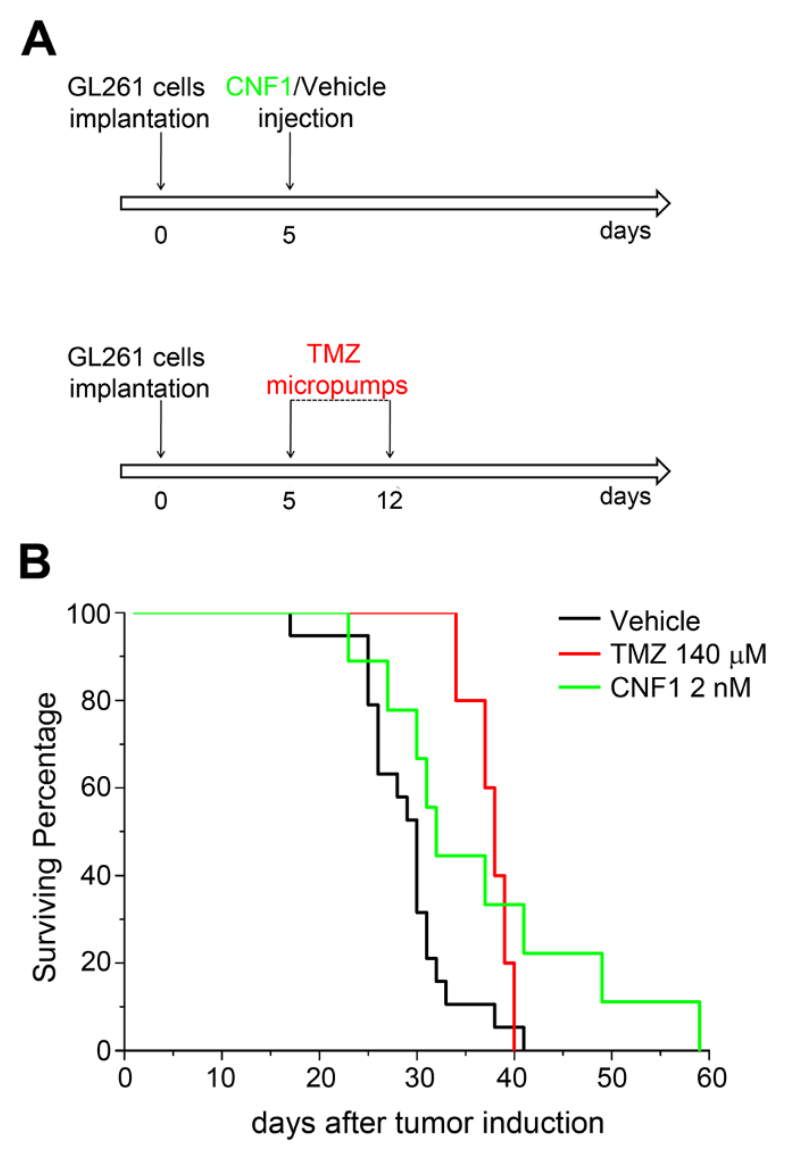

Figure 7 Comparison of the survival-promoting effects of CNF1 and TMZ in the GL261 glioma model. (A) Experimental protocol. Tumor induction by GL261 cells was either followed by a single intracerebral injection of CNF1/vehicle at day 5 (top) or by minipump delivery of TMZ from day 5 to day 12 (bottom). (B) Kaplan-Meier survival curves for animals implanted with GL261 cells. Compared to vehicle-infused mice (black line), CNF1 (green) and TMZ treatment (red) significantly increased survival (log Rank test followed by Holm-Sidak test, $\mathrm{p}<0.05$ ).

effect. Thus, even a short CNF1 exposure produced nearly maximal effects on cell proliferation. This may be important for glioma therapy, as one problem of local therapies is the rapid washout of therapeutic molecules infused in the tumor area. Moreover, the effects of CNF1 appear to persist for weeks following one single administration of the toxin $[10,11,30]$, likely due to persistent catalytic activity of the toxin inside cells. This further strengthens the potential of CNF1 and could avoid the need for repeated drug administration.

The potential involvement of CNF1 in cell transformation is still controversial [31]. Several studies in vitro and in vivo, including the present results, demonstrate the anti-proliferative and cytotoxic effect of CNF1 in cancer cell lines $[22,25,26]$. Furthermore, cell transformation and tumor formation have never been observed 


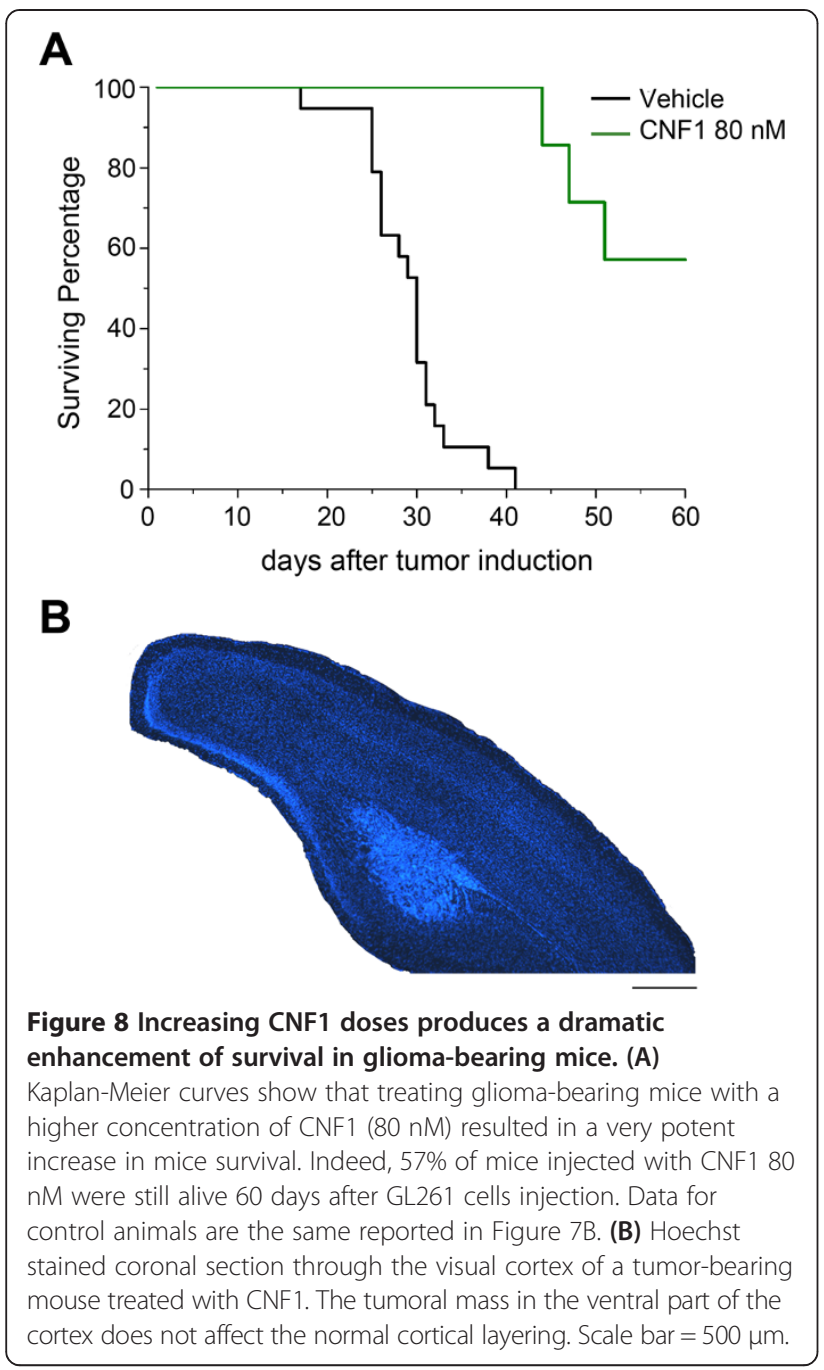

after a single administration of CNF1 in the rodent brain (e.g. $[10,11,30])$.

In order to evaluate the effectivness of CNF1, we compared its action to that of a current standard chemotherapic drug, namely TMZ. Even if TMZ in experimental animal models is typically given orally, here we have chosen to administer it via an intracranial route to allow direct comparison with CNF1, which does not cross the blood-brain barrier. We found that TMZ, administered for one week via minipumps, was effective in prolonging animal survival but had a quite narrow therapeutic range, with concentrations of $20 \mu \mathrm{M}$ being ineffective and concentrations > $200 \mu \mathrm{M}$ being toxic for the animals. The limited efficacy of TMZ chemotherapy is in line with current clinical and experimental experience $[4,32]$ and can be attributed to both inherent and acquired tumor drug resistance. In contrast with conventional chemotherapy, CNF1 had greater efficacy and showed no obvious side effects with increasing doses. Remarkably, more than half of the animals treated with
80 nM CNF1 survived for at least 60 days following glioma cell inoculation. One important aspect of CNF1 is its long-lasting action, as one single intracerebral administration leads to Rho GTPase activation for at least $10-28$ days $[10,11,30]$.

\section{Conclusions}

In summary, we have exploited the remarkable properties of CNF1 for interfering with glioma proliferation in vitro and in vivo. Specifically, we demonstrated in vitro that: (i) CNF1 blocks proliferation of GL261 cells, (ii) induces cell senescence and death, (ii) inhibits migration of tumor cells in the wound assay. In vivo, CNF1 was more effective than TMZ in prolonging survival of tumor-bearing mice. Given these antitumoral actions, and the ability of CNF1 to enhance learning, memory and plasticity in the intact and diseased brain $[10,11,30]$, the present data suggest that CNF1 might hinder glioma growth and at the same time preserve neuronal responses in the peritumoral area.

\section{Abbreviations}

CNF1: Cytotoxic necrotizing factor 1; TMZ: Temozolomide; GBM:

Glioblastoma multiforme; WHO: World Health Organization; ANOVA: Analysis of variance; SEM: Standard error of the mean; SA- $\beta$-gal: Senescence-associated $\beta$-galactosidase; LPS: Lipopolysaccharide.

\section{Competing interest}

The authors declare that they have no competing interest.

\section{Authors' contributions}

EV carried out all in vivo experiments, performed the statistical analysis and drafted the manuscript. AP carried out all in vitro experiments, performed the statistical analysis and drafted the manuscript. CC partecipated in the in vivo experiments. SL performed the in vitro experiments with human glioblastoma cells. EC performed the cell death assays. AF and CF provided CNF1 toxin. NB and RV provided human biopsies of glioblastoma multiforme. MCa and MCo conceived of the study, and participated in its design and coordination and drafted the manuscript. All authors read and approved the final manuscript.

\section{Authors' information}

Matteo Caleo and Mario Costa are equal contributors as senior authors.

\section{Acknowledgment}

This work was supported by AIRC (Italian Association for Cancer Research) grant \# IG 13252 and NanoBrain project (part of the CNR Flagship Project NanoMax).

\section{Author details}

${ }^{1}$ CNR Neuroscience Institute, Via Moruzzi 1, 56124 Pisa, Italy. ${ }^{2}$ Scuola Normale Superiore, Piazza Dei Cavalieri 7, 56100 Pisa, Italy. ${ }^{3}$ Istituto Superiore di Sanità, Viale Regina Elena 299, 00161 Rome, Italy. ${ }^{4}$ Neurochirurgia, Azienda Ospedaliero-Universitaria Pisana, Via Paradisa 2, 56100 Pisa, Italy.

Received: 22 January 2014 Accepted: 11 June 2014

Published: 18 June 2014

\section{References}

1. Khasraw M, Lassman AB: Advances in the treatment of malignant gliomas. Curr Oncol Rep 2010, 12(1):26-33.

2. Stupp R, Hegi ME, Gilbert MR, Chakravarti A: Chemoradiotherapy in malignant glioma: standard of care and future directions. J Clin Oncol 2007, 25(26):4127-4136.

3. King GD, Curtin JF, Candolfi M, Kroeger K, Lowenstein PR, Castro MG: Gene therapy and targeted toxins for glioma. Curr Gene Ther 2005, 5(6):535-557. 
4. Stupp R, Mason WP, van den Bent MJ, Weller M, Fisher B, Taphoorn MJ, Belanger K, Brandes AA, Marosi C, Bogdahn U, Curschmann J, Janzer RC, Ludwin SK, Gorlia T, Allgeier A, Lacombe D, Cairncross JG, Eisenhauer E, Mirimanoff RO: Radiotherapy plus concomitant and adjuvant temozolomide for glioblastoma. N Engl J Med 2005, 352(10):987-996.

5. Etienne-Manneville S, Hall A: Rho GTPases in cell biology. Nature 2002, 420(6916):629-635

6. Schmidt G, Sehr P, Wilm M, Selzer J, Mann M, Aktories K: Gln 63 of Rho is deamidated by Escherichia coli cytotoxic necrotizing factor-1. Nature 1997, 387(6634):725-729.

7. Flatau G, Lemichez E, Gauthier M, Chardin P, Paris S, Fiorentini C, Boquet P: Toxin-induced activation of the $\mathrm{G}$ protein p21 Rho by deamidation of glutamine. Nature 1997, 387(6634):729-733.

8. Doye A, Mettouchi A, Bossis G, Clement R, Buisson-Touati C, Flatau G, Gagnoux L, Piechaczyk M, Boquet P, Lemichez E: CNF1 exploits the ubiquitin-proteasome machinery to restrict Rho GTPase activation for bacterial host cell invasion. Cell 2002, 111(4):553-564.

9. Falzano L, Fiorentini C, Boquet P, Donelli G: Interaction of Escherichia coli cytotoxic necrotizing factor type 1 (CNF1) with cultured cells. Cytotechnology 1993, 11(Suppl 1):S56-58.

10. Cerri C, Fabbri A, Vannini E, Spolidoro M, Costa M, Maffei L, Fiorentini C, Caleo M: Activation of Rho GTPases triggers structural remodeling and functional plasticity in the adult rat visual cortex. J Neurosci 2011, 31(42):15163-15172.

11. Diana G, Valentini G, Travaglione S, Falzano L, Pieri M, Zona C, Meschini S, Fabbri A, Fiorentini C: Enhancement of learning and memory after activation of cerebral Rho GTPases. Proc Natl Acad Sci U S A 2007, 104(2):636-641.

12. Zagzag D, Miller DC, Chiriboga L, Yee H, Newcomb EW: Green fluorescent protein immunohistochemistry as a novel experimental tool for the detection of glioma cell invasion in vivo. Brain Pathol 2003, 13(1):34-37.

13. Miyatake S, Martuza RL, Rabkin SD: Defective herpes simplex virus vectors expressing thymidine kinase for the treatment of malignant glioma. Cancer Gene Ther 1997, 4(4):222-228.

14. Falzano L, Fiorentini C, Donelli G, Michel E, Kocks C, Cossart P, Cabanie L, Oswald $E$, Boquet $P$ : Induction of phagocytic behaviour in human epithelial cells by Escherichia coli cytotoxic necrotizing factor type 1. Mol Microbiol 1993, 9(6):1247-1254.

15. Franken NA, Rodermond HM, Stap J, Haveman J, van Bree C: Clonogenic assay of cells in vitro. Nat Protoc 2006, 1(5):2315-2319.

16. Liang CC, Park AY, Guan JL: In vitro scratch assay: a convenient and inexpensive method for analysis of cell migration in vitro. Nat Protoc 2007, 2(2):329-333.

17. Liau LM, Lallone RL, Seitz RS, Buznikov A, Gregg JP, Kornblum HI, Nelson SF, Bronstein JM: Identification of a human glioma-associated growth factor gene, granulin, using differential immuno-absorption. Cancer Res 2000 60(5):1353-1360

18. Day BW, Stringer BW, Al-Ejeh F, Ting MJ, Wilson J, Ensbey KS, Jamieson PR, Bruce ZC, Lim YC, Offenhauser C, Charmsaz S, Cooper LT, Ellacott JK, Harding A, Leveque L, Inglis P, Allan S, Walker DG, Lackmann M, Osborne G, Khanna KK, Reynolds BA, Lickliter JD, Boyd AW: EphA3 maintains tumorigenicity and is a therapeutic target in glioblastoma multiforme. Cancer Cell 2013, 23(2):238-248.

19. Restani L, Cerri C, Pietrasanta M, Gianfranceschi L, Maffei L, Caleo M: Functional masking of deprived eye responses by callosal input during ocular dominance plasticity. Neuron 2009, 64(5):707-718.

20. Lodovichi C, Berardi N, Pizzorusso T, Maffei L: Effects of neurotrophins on cortical plasticity: same or different? J Neurosci 2000, 20(6):2155-2165.

21. Li YM, Hall WA: Targeted toxins in brain tumor therapy. Toxins (Basel) 2010, 2(11):2645-2662.

22. Fabbri A, Travaglione S, Fiorentini C: Escherichia coli cytotoxic necrotizing factor 1 (CNF1): toxin biology, in vivo applications and therapeutic potential. Toxins (Basel) 2010, 2(2):283-296.

23. Aktories K: Bacterial protein toxins that modify host regulatory GTPases. Nat Rev Microbiol 2011, 9(7):487-498.

24. Fiorentini C, Fabbri A, Flatau G, Donelli G, Matarrese P, Lemichez E, Falzano $L$, Boquet P: Escherichia coli cytotoxic necrotizing factor 1 (CNF1), a toxin that activates the Rho GTPase. J Biol Chem 1997, 272(31):19532-19537.

25. Caprioli A, Falbo V, Roda LG, Ruggeri FM, Zona C: Partial purification and characterization of an escherichia coli toxic factor that induces morphological cell alterations. Infect Immun 1983, 39(3):1300-1306.
26. Caprioli A, Donelli G, Falbo V, Possenti R, Roda LG, Roscetti G, Ruggeri FM: A cell division-active protein from E coli. Biochem Biophys Res Commun 1984, 118(2):587-593.

27. Magana-Maldonado R, Manoutcharian K, Hernandez-Pedro NY, RangelLopez E, Perez-Dela Cruz V, Rodriguez-Balderas C, Sotelo J, Pineda B: Concomitant treatment with pertussis toxin plus temozolomide increases the survival of rats bearing intracerebral RG2 glioma. J Cancer Res Clin Oncol 2014, 140(2):291-301.

28. Chicoine MR, Won EK, Zahner MC: Intratumoral injection of lipopolysaccharide causes regression of subcutaneously implanted mouse glioblastoma multiforme. Neurosurgery 2001, 48(3):607-614

29. Salhia B, Rutten F, Nakada M, Beaudry C, Berens M, Kwan A, Rutka JT: Inhibition of Rho-kinase affects astrocytoma morphology, motility, and invasion through activation of Rac1. Cancer Res 2005, 65(19):8792-8800.

30. De Filippis B, Fabbri A, Simone D, Canese R, Ricceri L, Malchiodi-Albedi F, Laviola G, Fiorentini C: Modulation of RhoGTPases improves the behavioral phenotype and reverses astrocytic deficits in a mouse model of Rett syndrome. Neuropsychopharmacology 2012, 37(5):1152-1163.

31. Travaglione S, Fabbri A, Fiorentini C: The Rho-activating CNF1 toxin from pathogenic E. coli: a risk factor for human cancer development? Infect Agent Cancer 2008, 3:4.

32. Ruban A, Berkutzki T, Cooper I, Mohar B, Teichberg VI: Blood glutamate scavengers prolong the survival of rats and mice with brain-implanted gliomas. Invest New Drugs 2012, 30(6):2226-2235.

doi:10.1186/1471-2407-14-449

Cite this article as: Vannini et al:: The bacterial protein toxin, cytotoxic necrotizing factor 1 (CNF1) provides long-term survival in a murine glioma model. BMC Cancer 2014 14:449.

\section{Submit your next manuscript to BioMed Central and take full advantage of:}

- Convenient online submission

- Thorough peer review

- No space constraints or color figure charges

- Immediate publication on acceptance

- Inclusion in PubMed, CAS, Scopus and Google Scholar

- Research which is freely available for redistribution

Submit your manuscript at www.biomedcentral.com/submit
C Biomed Central 